\title{
JETS OF CLOSED ORBITS OF MAÑÉ GENERIC HAMILTONIAN FLOWS
}

\author{
C. M. CARBALLO AND J. A. G. MIRANDA
}

\begin{abstract}
We prove a perturbation theorem for the $k$-jets, $k \geq 2$, of the Poincaré map of a closed orbit of the Hamiltonian flow of a Tonelli Hamiltonian $H: T^{*} M \rightarrow \mathbb{R}$, on a closed manifold $M$. As a consequence we obtain Mañé generic properties of Hamiltonian and Lagrangian flows.
\end{abstract}

\section{INTRODUCTION}

Let $M$ be a closed Riemannian manifold and $T^{*} M$ its cotangent bundle endowed with the canonical symplectic structure $\omega=-d \Theta$, where $\Theta$ is the Liouville 1-form. If $\pi: T^{*} M \rightarrow M$ is the canonical projection, $\Theta$ is defined by

$$
\Theta_{(x, p)}(\xi)=p\left(d_{(x, p)} \pi(\xi)\right), \text { for } \xi \in T_{(x, p)} T^{*} M .
$$

Let $H: T^{*} M \rightarrow \mathbb{R}$ be a Hamiltonian of class $C^{2}$ that is convex, i.e., for each fiber $T_{x}^{*} M$, the restriction $H(x, p)$ has positive defined Hessian, and superlinear, i.e., $\lim _{\|p\| \rightarrow \infty} \frac{H(x, p)}{\|p\|}=\infty$, uniformly in $x \in M$. A Hamiltonian like this is often called a Tonelli Hamiltonian. The Hamiltonian vector field $X_{H}$ of $H$ is defined by

$$
\omega\left(X_{H}, \cdot\right)=d H(\cdot)
$$

and the Hamiltonian flow, $\phi_{t}^{H}: T^{*} M \rightarrow T^{*} M$, of $H$ is the flow corresponding to $X_{H}$. Observe that this flow preserves the Hamiltonian function and the symplectic structure $\omega$. The subsets $H^{-1}(c) \subset T^{*} M$ are called energy levels of $H$. Then the compactness of $M$ and the superlinearity of $H$ imply that the nonempty energy levels are compact. Then the Hamiltonian flow is defined for all $t \in \mathbb{R}$.

The theory of Hamiltonian flows is closely related to that of the Lagrangian flows. This is the reason to restrict to Tonelli Hamiltonians or Lagrangians. Given a Tonelli, i.e., convex and superlinear, Lagrangian $L: T M \rightarrow \mathbb{R}$, of class $C^{3}$, the Lagrangian flow of $L$ is conjugated to a Hamiltonian flow in $T^{*} M$ by the Legendre transformation $\mathcal{F}_{L}: T M \rightarrow T^{*} M$, defined by

$$
\mathcal{F}_{L}(x, v)=\left(x, \frac{\partial L}{\partial v}(x, v)\right) .
$$


The corresponding convex Hamiltonian $H: T^{*} M \rightarrow \mathbb{R}$ is

$$
H(x, p)=\max _{v \in T_{x} M}\{p(v)-L(x, v)\} .
$$

Conversely, given a Hamiltonian $H: T^{*} M \rightarrow \mathbb{R}$, the corresponding Lagrangian, defined by the Legendre transformation of the Hamiltonian $\mathcal{F}_{H}$ : $T^{*} M \rightarrow T M$, is

$$
L(x, v)=\max _{p \in T_{x}^{*} M}\{p(v)-H(x, p)\} .
$$

In this paper we study generic properties, in the sense of Mañé, of the jets of closed orbits of Tonelli Hamiltonian flows. Let $C^{\infty}(M)$ be the space of smooth functions $u: M \rightarrow \mathbb{R}$ endowed with the $C^{\infty}$ topology. Recall that a subset $\mathcal{O} \subset C^{\infty}(M)$ is called residual if it contains a countable intersection of open and dense subsets. We say that a property is Mañé generic, if for each Lagrangian $L: T M \rightarrow \mathbb{R}$, there exists a residual subset $\mathcal{O} \subset C^{\infty}(M)$, such that the property holds for all $L_{u}=L-u$, with $u \in \mathcal{O}$. The corresponding definition in the Hamiltonian setting is obtained considering perturbations $H_{u}=H+u$.

This genericity concept was introduced by R. Mañé in [Mañ96] to study Aubry-Mather theory (see [Mat91]) for Lagrangian systems and, after this, many works were devoted to studying generic properties of Lagrangian and Hamiltonian systems in the sense of Mañé; see, for instance, [CI99, CP02, Mas03, BC08].

Also in this context, E. Oliveira, in [Oli08], proved a version of the KupkaSmale theorem for generic Hamiltonians on surfaces. In [RR11], L. Rifford and R. Ruggiero proved a perturbation theorem of the 1-jet of the Poincaré map of a closed orbit for Hamiltonians on a compact manifold of any dimension and, using this perturbation theorem and Oliveira's results, obtained a version of the Kupka-Smale theorem for Tonelli Hamiltonians and Lagrangians on closed manifolds of any dimension.

Theorem 1 (Kupka-Smale). Let $M$ be a closed manifold and $H: T^{*} M \rightarrow \mathbb{R}$ a $C^{2}$ Tonelli Hamiltonian. Then, for each $c \in \mathbb{R}$, there exists a residual set $\mathcal{K S}=\mathcal{K S}(c) \subset C^{\infty}(M)$ such that every Hamiltonian $H_{u}=H+u$, with $u \in \mathcal{K S}$, satisfies

(1) $H_{u}^{-1}(c)$ is a regular energy level,

(2) all closed orbits in $H_{u}^{-1}(c)$ are non-degenerate, and

(3) all heteroclinic intersections in $H_{u}^{-1}(c)$ are transverse.

The main goal of the present paper is to complete this result to include conditions on the higher order derivatives of the Poincaré maps of closed orbits. It is motivated by the fact that the dynamical behavior near elliptic closed orbits depend on the higher order derivatives of the corresponding Poincaré map (see Corollary 5). 
What we do here is analogous to what has been done for other classes of conservative systems. Let us consider, for instance, the geodesic flows, and recall that a bumpy metric is a metric such that all closed geodesics are non-degenerate. The bumpy metric theorem states that the subset of bumpy metrics is a residual subset of the space of smooth Riemannian metrics on $M$. This theorem is attributed to R. Abraham [Abr70]; see also D. V. Anosov [Ano82], where a complete proof is given. In [KT72], Klingenberg and Takens extend the bumpy metric theorem to include conditions on the $k$-jets of the Poincaré map over closed orbits for geodesic flows. For the class of magnetic flows on surfaces, a complete study of generic properties of closed orbits can be found in [Mir06].

Let us recall some facts about the jet space of symplectic diffeomorphisms. We consider the space of smooth diffeomorphisms $f: \mathbb{R}^{m} \rightarrow \mathbb{R}^{m}$ such that $f(0)=0$. Given $k \in \mathbb{N}$, we say that the diffeomorphisms $f$ and $g$ are $k$ equivalent if their Taylor polynomials of degree $k$ at 0 are equal. The $k$-jet of a diffeomorphism $f$ at $0, j^{k} f(0)$, or $j^{k} f$ for short, is the equivalence class of $f$. If we consider only symplectic diffeomorphisms in $\mathbb{R}^{2 n}$, the set of all the equivalence classes is the space of symplectic $k$-jets, that we denote $J_{s}^{k}(n)$. Observe that $J_{s}^{k}(n)$ is a vector space and that it is also a Lie group, with the product defined by

$$
j^{k} f \cdot j^{k} g=j^{k}(f \circ g) .
$$

We say that a subset $Q \subset J_{s}^{k}(n)$ is invariant if

$$
\sigma \cdot Q \cdot \sigma^{-1}=Q, \forall \sigma \in J_{s}^{k}(n) .
$$

Note that if $M$ has dimension $n+1, \theta$ is a nontrivial closed orbit of the Hamiltonian flow of $H: T^{*} M \rightarrow \mathbb{R}$ in the energy level $H^{-1}(c)$, and $\Sigma \subset H^{-1}(c)$ is a local transverse section at the point $\theta(0)$, then $\Sigma$ also has a symplectic structure and the Poincaré map $P(\theta(0), \Sigma, L): \Sigma \rightarrow \Sigma$ is a symplectic diffeomorphism. Therefore, using Darboux coordinates, we can assume that $\left.j^{k} P(\theta(0), \Sigma, L)\right) \in J_{s}^{k}(n)$.

Given an invariant subset $Q \subset J_{s}^{k}(n)$ and a closed orbit $\theta$ of a Hamiltonian flow, it follows from (1) that the property "the $k$-jet of the Poincaré map of $\theta$ belongs to $Q$ " is independent of the section $\Sigma$ and the coordinate system; hence, it is well defined.

Using the notation above, we state our perturbation theorem. Recall that, given a Tonelli Hamiltonian $H$, we denote the perturbed Hamiltonian $H_{u}=$ $H+u$.

Theorem 2. Let $M$ be a closed manifold of dimension $n+1, H: T^{*} M \rightarrow$ $\mathbb{R}, k \geq 1$, and $Q$ any open invariant subset of $J_{s}^{k}(n)$. Suppose that $\theta(t)=$ $(\gamma(t), p(t))$ is a nontrivial closed orbit of the Hamiltonian flow of $H$. If the $k$-jet of the Poincaré map of $\theta$ is in $\bar{Q}$, then there exists a smooth potential $u: M \rightarrow \mathbb{R}, C^{r}$-close to zero, $r \geq k+1$, such that 
(1) $\theta(t)$ is also a closed orbit of the Hamiltonian flow of $H_{u}$ and

(2) the $k$-jet of the Poincaré map of $\theta$, as a closed orbit of the Hamiltonian flow of $H_{u}$, is in $Q$.

In the particular case $k=1$ the theorem was proved by L. Rifford and R. Ruggiero: Proposition 4.2 and Lemma 4.3 in [RR11] show that an open set of $S p(n)=J_{s}^{1}(n)$ is attained by a family of differentials of the Poincaré maps of the closed orbit $\theta$ obtained by perturbing the initial Hamiltonian $H$ by adding small potentials. See also [Oli08, Theorem 4.5]. Our contribution is the case of higher order jets, $k \geq 2$.

Combining the Kupka-Smale theorem stated above, Theorem 1, and our perturbation theorem, we obtain the genericity result.

Theorem 3. Let $M$ be a closed manifold of dimension $n+1$. For every open, dense, and invariant subset $Q \subset J_{s}^{k}(n)$ and every $c \in \mathbb{R}$, there is a residual subset $\mathcal{O}=\mathcal{O}(Q, c)$ of $C^{\infty}(M)$ such that, if $u \in \mathcal{O}$, then the $k$-jet of the Poincaré map of any closed orbit of the Hamiltonian flow of $H_{u}=H+u$ in $H_{u}^{-1}(c)$ is in $Q$.

One needs Theorem 3 to study the dynamics of a Mañé generic Hamiltonian flow that has a non-hyperbolic closed orbit in a regular energy level. Recall that a closed orbit $\theta$ in $H^{-1}(c)$ is q-elliptic if the derivative of its Poincaré map $P$ has exactly $2 q$ eigenvalues of modulus 1 which are non-real; it is quasielliptic if it is $q$-elliptic for some $q>0$. If $\theta$ is a $q$-elliptic closed orbit, then the central manifold $W^{c}$ of the Poincaré map $P: \Sigma \rightarrow \Sigma$ of $\theta$ has dimension $2 q$, $\left.\omega\right|_{W^{c}}$ is non-degenerate, and $F=\left.P\right|_{W^{c}}$ is a symplectic map on a sufficiently small neighborhood of $\theta$.

Let $\lambda_{1}, \ldots, \lambda_{q}, \overline{\lambda_{1}}, \ldots, \overline{\lambda_{q}}$ be the eigenvalues of modulus 1 of the derivative $d_{\theta} P$ of the Poincaré map of a $q$-elliptic orbit $\theta$. We say that $\theta$ is 4 -elementary if $\prod_{i=1}^{q} \lambda_{i}^{m_{i}} \neq 1$ whenever the integers $m_{i}$ satisfy $1 \leq \sum_{i=1}^{q}\left|m_{i}\right| \leq 4$.

Theorem 4 (Birkhoff Normal Form). Let $F: \mathbb{R}^{2 q} \rightarrow \mathbb{R}^{2 q}$ be a symplectic diffeomorphism such that 0 is a q-elliptic 4-elementary fixed point for $F$.

Then there are symplectic coordinates $(x, y)=\left(x_{1}, \ldots, x_{q}, y_{1}, \ldots, y_{q}\right)$ in a neighborhood of 0 such that

$$
F_{k}(x, y)=F_{k}(z)=e^{2 \pi i \phi_{k}(z)} z_{k}+R_{k}(z), \quad k=1, \ldots, q,
$$

where $z=x+i y, \phi_{k}(z)=a_{k}+\sum_{l=1}^{q} \beta_{k l}\left|z_{l}\right|^{2}, \lambda_{k}=e^{2 \pi i a_{k}}$, and $R_{k}(z)$ has zero derivatives up to order 3 at 0 .

For a proof, see [Kli78, p. 101].

Then, we say that a $q$-elliptic closed orbit $\theta$ is weakly monotonous if the coefficients $\beta_{k l}$ of $F=\left.P\right|_{W^{c}}$ satisfy $\operatorname{det}\left[\beta_{k l}\right] \neq 0$.

Let $Q \subset J_{s}^{3}(n)$ be the set of 3 -jets of $C^{\infty}$ symplectic diffeomorphisms $T$ of $\mathbb{R}^{2 n}$ such that the origin is

(1) a hyperbolic fixed point or 
(2) a weakly monotonous 4-elementary $q$-elliptic fixed point, for some $q>0$.

Observe that $Q$ is open, dense and invariant; recall the definition of invariant, equation (1). Let $c \in \mathbb{R}$ define the energy level. By applying Theorem 3, to $Q$ and $c$, we get a residual subset $\mathcal{O}$. Now, if $u \in \mathcal{O}$ and $\theta$ is a non-hyperbolic orbit of $H_{u}$ in the energy level $H_{u}^{-1}(c)$, then the 3-jet of the Poincaré map of $\theta$ is in $Q$. Hence, $\theta$ is a weakly monotonous $q$-elliptic orbit, for some $q>1$. This proves the following result.

Corollary 5. Let $M$ be a closed manifold, $H: T^{*} M \rightarrow \mathbb{R}$ a Tonelli Hamiltonian and $c \in \mathbb{R}$. There is a residual subset $\mathcal{O}=\mathcal{O}(c)$ of $C^{\infty}(M)$ such that, for every $u \in \mathcal{O}$, any closed orbit of $H_{u}$ in $H_{u}^{-1}(c)$ is either hyperbolic or weakly monotonous quasi-elliptic.

Observe that what we called here weakly monotonous is a generalization of the twist condition of surface diffeomorphisms. This property has important dynamical consequences such as the existence of a nontrivial hyperbolic set and then, infinitely many periodic orbits and positive topological entropy. We refer to [Con10] for the details. In this paper G. Contreras treats the case of geodesic flows but the arguments apply to our context as well.

\section{The $k$-General POSITION}

In this preliminary section, we introduce the idea of $k$-general position through an open and dense set $G_{k}$. Later we will use this concept to perform the perturbation of the jets.

We identify $J_{s}^{1}(n)$ with the classic Lie group $S p(n)$ of symplectic maps in $\mathbb{R}^{2 n}$ which is also identified with the group of $2 n \times 2 n$ symplectic matrices, i.e., with the matrices that satisfy $\sigma^{T} J \sigma=J$, where

$$
J=\left[\begin{array}{cc}
0 & I \\
-I & 0
\end{array}\right]
$$

and $I=I_{n}$ is the $n \times n$ identity matrix.

For each $k \in \mathbb{N}$, we denote by $\mathbb{R}_{k}[x, y]$ the set of all real homogeneous polynomials of degree $k$ in the $2 n$ variables $x=\left(x_{1}, \ldots, x_{n}\right), y=\left(y_{1}, \ldots, y_{n}\right)$. This is a real vector space of dimension $d=d(2 n, k)=\left(\begin{array}{c}2 n-1+k \\ k\end{array}\right)$.

We fix the polynomial $F\left(x_{1}, \ldots, x_{n}, y_{1}, \ldots, y_{n}\right)=x_{1}^{k}$ and we define

$$
G_{k}=\left\{\left(\sigma_{1}, \ldots, \sigma_{d}\right) \in S p(n)^{d}:\left\{F \circ \sigma_{1}, \ldots, F \circ \sigma_{d}\right\} \text { is a basis of } \mathbb{R}_{k}[x, y]\right\} \text {. }
$$

Proposition 6. For each $k \in \mathbb{N}$, the subset $G_{k}$ is open and dense in $S p(n)^{d}$.

Proof. Let $F_{1}, \ldots, F_{d}$ be a basis of the vector space $\mathbb{R}_{k}[x, y]$ and define the matrix $A\left(\sigma_{1}, \ldots, \sigma_{d}\right)=\left[a_{i j}\right]$ by the equation $F \circ \sigma_{j}=\sum_{i} a_{i j} F_{i}$. Then $G_{k}$ is the complement in $S p(n)^{d}$ of the set

$$
\left\{\left(\sigma_{1}, \ldots, \sigma_{d}\right) \in S p(n)^{d}: \operatorname{det} A\left(\sigma_{1}, \ldots, \sigma_{d}\right)=0\right\} .
$$


This shows that $G_{k}$ is the complement of an algebraic subset.

To verify that $G_{k}$ is non-empty, let us consider the following example. Define

$$
\sigma=\left[\begin{array}{cc}
A & B \\
0 & \left(A^{-1}\right)^{T}
\end{array}\right]
$$

where

$$
A=\left[\begin{array}{c|c}
1 & a_{2} \ldots a_{n} \\
-- & ------ \\
0 & \\
\vdots & I_{n-1}
\end{array}\right], \quad B=\left[\begin{array}{c|c}
a_{n+1} & a_{n+2} \ldots a_{2 n} \\
-- & ------ \\
b_{2} & \\
\vdots &
\end{array}\right]
$$

and $b_{i}=a_{n+i}-a_{i}$, for $i=2, \ldots, n$. It is easy to check that the matrix $A^{-1} B$ is symmetric; hence, $\sigma$ is symplectic.

Then the composition $F \circ \sigma$ defines the homogeneous polynomials

$$
\left(x_{1}+a_{2} x_{2}+\cdots+a_{n} x_{n}+a_{n+1} y_{1}+\cdots+a_{2 n} y_{n}\right)^{k} .
$$

Let us show that there exist values $a_{i j}$ such that the corresponding set of polynomials contain a basis of $\mathbb{R}_{k}[x, y]$. To simplify the notation we write the argument using arbitrary variables $x_{1}, \ldots, x_{m}$. First, observe that

$$
\left(x_{1}+a_{2} x_{2}+\cdots+a_{m} x_{m}\right)^{k}=\sum_{\alpha_{1}+\cdots+\alpha_{m}=k} a_{2}^{\alpha_{2}} \cdots a_{m}^{\alpha_{m}} c_{\alpha_{1} \cdots \alpha_{m}} x_{1}^{\alpha_{1}} \cdots x_{m}^{\alpha_{m}},
$$

where all the coefficients $c_{\alpha_{1} \cdots \alpha_{m}}$ are different from zero so that the monomials $c_{\alpha_{1} \cdots \alpha_{m}} x_{1}^{\alpha_{1}} \cdots x_{m}^{\alpha_{m}}$ are a basis of the space of homogeneous polynomials of degree $k$. Then, we let $a_{i}=t^{p_{i}}$ and need to choose the powers $p_{i}$ to ensure that after this replacement we have different powers of $t$ in all the $a_{2}^{\alpha_{2}} \cdots a_{m}^{\alpha_{m}}$. One can check that a possible choice is $p_{i}=\sum_{j=0}^{i-2} k^{j}, i=2, \ldots, m$. Now that the scalars are all powers of $t$, the result follows using the result about Vandermonde matrices.

\section{Proof of Theorem 2}

Let $\theta=\theta(t)=(\gamma(t), p(t))$ be a closed orbit of the Hamiltonian flow of the initial Hamiltonian $H_{0}=H: T^{*} M \rightarrow \mathbb{R}$ and let $T>0$ be its minimal period. Recall that, by the Kupka-Smale Theorem, we can assume that $\theta$ is a non-degenerate closed orbit and that the energy level $H_{0}^{-1}(c)$ that contains $\theta$ is regular.

Since the number of self-intersection points is finite, we can choose a time $a \in(0, T]$, such that the arc $\gamma([0, a])$ does not contain self-intersection points of the curve $\gamma$ and a tubular neighborhood $W \subset M$ of $\gamma([0, a])$, sufficiently small, such that $W \cap \gamma([0, T])=\gamma([0, a])$. 
Then, we choose local coordinates $\left(x_{0}, x\right): W \rightarrow \mathbb{R} \times \mathbb{R}^{n}, x=\left(x_{1}, \ldots, x_{n}\right)$, such that

$$
x_{0}(\gamma(t))=t \quad \text { and } \quad x(\gamma(t))=(0, \ldots, 0),
$$

for $t \in[0, a]$. Then, if $p \in T_{\left(x_{0}, x\right)}^{*} M$, we define $y_{i}$ by $p=\sum y_{i} d x_{i}$ and we have a natural chart

$$
\left(x_{0}, x, y_{0}, y\right)=\left(x_{0}, x_{1}, \ldots, x_{n}, y_{0}, y_{1}, \ldots, y_{n}\right)
$$

of $\pi^{-1}(W) \subset T^{*} M$.

In these coordinates we have that the 2 -form $\omega$ that defines the symplectic structure in $T^{*} M$ can be written $\omega=\sum_{i=0}^{n} d x_{i} \wedge d y_{i}$ and that the Hamiltonian vector field $X_{H}$ of a Hamiltonian $H$ is

$$
X_{H}=\sum_{i=0}^{n}\left(\frac{\partial H}{\partial y_{i}} \frac{\partial}{\partial x_{i}}-\frac{\partial H}{\partial x_{i}} \frac{\partial}{\partial y_{i}}\right)
$$

Now, we define our perturbation space.

Given $k \in \mathbb{N}$, let $\mathcal{F}^{k}=\mathcal{F}\left(W, \gamma, H_{0}, a, k\right)$ be the subset of smooth functions $u: M \rightarrow \mathbb{R}$ supported in $W$ such that, in the local coordinates $\left(x_{0}, x\right)$, have the form

$$
u\left(x_{0}, x_{1}, \ldots, x_{n}\right)=\delta\left(x_{0}\right) \beta\left(x_{1}, \ldots, x_{n}\right),
$$

where $\delta: \mathbb{R} \rightarrow \mathbb{R}$ and $\beta: \mathbb{R}^{n} \rightarrow \mathbb{R}$ are smooth functions satisfying

- $\operatorname{supp}(\delta) \subset(0, a)$

- $\operatorname{supp}(\beta) \subset B_{\epsilon}(0)$, with $\epsilon$ sufficiently small, and

- $j^{k+1} \beta(0)$ is homogeneous of degree $k+1$.

We will consider perturbations $H_{u}=H+u$ of the initial Hamiltonian $H_{0}=H$, with $u \in \mathcal{F}^{k} \subset C^{\infty}(M)$.

Then $X_{H_{u}}=X_{H_{0}}+X_{u}$ and, by (2), we have

$$
X_{u}\left(x_{0}, x, y_{0}, y\right)=-\delta^{\prime}\left(x_{0}\right) \beta(x) \frac{\partial}{\partial y_{0}}-\sum_{i=1}^{n} \delta\left(x_{0}\right) \frac{\partial \beta}{\partial x_{i}}(x) \frac{\partial}{\partial y_{i}} .
$$

Note that, if $u \in \mathcal{F}^{k}$, then the closed orbit $\theta$ is still a closed orbit of the Hamiltonian flow of $H_{u}$

Using the coordinates $\left(x_{0}, x, y_{0}, y\right)$ in $\pi^{-1}(W) \subset T^{*} M$, we set $\Lambda(t)=\left\{x_{0}=\right.$ $t\}$. This defines a family of local hypersurfaces in $T^{*} M$ that are transverse to $\theta(t)$, for $t \in[0, a]$. It follows from the definition of the set $\mathcal{F}^{k}$ that the vector field $X_{u}$ satisfy

- $j^{k-1} X_{u}(\theta(t))=0$, for all $t \in[0, a]$,

- neither $\theta(0)$ nor $\theta(a)$ is in $\operatorname{supp}\left(X_{u}\right)$, and

- $\left.X_{u}\right|_{\Lambda(t)}$ is tangent to $\Lambda(t)$, for all $t \in[0, a]$.

Also, for each $t \in[0, a]$, we denote

$$
R_{u, t}=\widehat{P}_{0, t}^{-1} \circ \widehat{P}_{u, t},
$$


where $\widehat{P}_{0, t}: \Lambda(0) \rightarrow \Lambda(t)$ and $\widehat{P}_{u, t}: \Lambda(0) \rightarrow \Lambda(t)$ are the Poincaré maps in an open neighborhood of $\theta(0) \in \Lambda(0)$ with respect to $H_{0}$ and $H_{u}$ respectively.

The following proposition holds for abstract vector fields satisfying the three conditions above. For a proof, see [KT72, section 2].

Proposition 7. The $k$-jet of $R_{u, a}$ at $\theta(0)$ is equal to the $k$-jet of the time-a map of the flow of the non autonomous vector field $\widehat{P}_{0, t}^{*}\left(\left.X_{u}\right|_{\Lambda(t)}\right)$ at $\theta(0)$.

We are interested in the $k$-jet of the map $R_{u, a}$ restricted to the energy level $H_{0}^{-1}(c)$. First, note that the energy levels $H_{0}^{-1}(c)$ and $H_{u}^{-1}(c)$ are $k$-tangent along the closed orbit $\theta$, for all $u \in \mathcal{F}^{k}$.

For each $u \in \mathcal{F}^{k}$, let $\Sigma_{u}(t) \subset T^{*} M$ be the submanifold given by $\Sigma_{u}(t)=$ $\Lambda(t) \cap H_{u}^{-1}(c)$. Then $\omega$ induces a symplectic structure on $\Sigma(t)$ and the restriction $\left.\widehat{P}_{0, t}\right|_{\Sigma_{0}(0)}: \Sigma_{0}(0) \rightarrow \Sigma_{0}(t)$ is a symplectic map for all $t \in[0, a]$. Since neither $\theta(0)$ nor $\theta(a)$ is in $\operatorname{supp}\left(X_{u}\right)$, the restriction $\left.\widehat{P}_{u, a}\right|_{\Sigma_{0}(0)}: \Sigma_{0}(0) \rightarrow \Sigma_{0}(a)$ is a symplectic map too.

Observe that $\frac{\partial H_{0}}{\partial y_{0}}(\theta(t)) \equiv 1$. Then we can parameterize $\Sigma_{0}(t)$ in terms of the coordinates $(x, y)$, this is, for each $t \in[0, a]$ there is an open set $V_{t} \subset \mathbb{R}^{2 n}$ and a function $\alpha_{t}: V_{t} \rightarrow \mathbb{R}$, such that

$$
\Sigma_{0}(t)=\left\{\left(t, x, \alpha_{t}(x, y), y\right) \in \Lambda(t):(x, y) \in V_{t}\right\} .
$$

Since $T \Sigma_{0}(t) \subset \operatorname{ker}\left(d x_{0}\right)$, the symplectic structure induced by $\omega$ in $\Sigma_{0}(t)$ is given by $\left.\omega\right|_{\Sigma_{0}(t)}=d x \wedge d y$. For each $u \in \mathcal{F}^{k}$ and $t \in[0, a]$, we consider the Hamiltonian function $K_{u, t}: \Sigma_{0}(t) \rightarrow \mathbb{R}$ given by

$$
K_{u, t}=\left.u\right|_{\Sigma_{0}(t)}=\delta(t) \beta\left(x_{1}, \ldots, x_{n}\right)
$$

and we denote by $Y_{u, t}$ its Hamiltonian vector field.

Then

$$
j^{k+1} K_{u, t}(\theta(t))=\delta(t) x_{1}^{k+1}
$$

and this defines a family, parameterized by $t$, of multiples of the polynomial

$$
F\left(x_{1}, \ldots, x_{n}, y_{1}, \ldots, y_{n}\right)=x_{1}^{k+1} .
$$

Remark 8. If $(t, x) \in \operatorname{supp}(u)$, the submanifold $\Sigma_{0}(0)$ is not invariant by the map $R_{u, t}$. But, the $k$-jet of the component of $X_{u}$ in the direction of $\frac{\partial}{\partial y_{0}}$ at $\theta(t)$ is zero, see $(3)$, and the directions $\frac{\partial}{\partial y_{i}}$, for all $i=1, \ldots, n$, are tangent to $\Sigma_{0}(t)$ along $\theta(t)$, because $d_{\theta(t)} H\left(\frac{\partial}{\partial y_{i}}\right)=\omega_{\theta(t)}\left(X_{H_{0}}, \frac{\partial}{\partial y_{i}}\right) \equiv 0$. Then the vector fields $Y_{u, t}$ and $\left.X_{u}\right|_{\Sigma_{0}(t)}$ in $\Sigma_{0}(t)$ have the same $k$-jet along $\theta(t)$. Moreover, since $\widehat{P}_{0, t}\left(\Sigma_{0}(0)\right)=\Sigma_{0}(t)$, we have that the non autonomous vector field $\widehat{P}_{0, t}^{*}\left(\left.X_{u}\right|_{\Sigma_{0}(t)}\right)$ is $k$-tangent to $\Sigma_{0}(0) \subset \Lambda(0)$. Therefore the submanifolds $\Sigma_{0}(0)$ and $R_{u, t}\left(\Sigma_{0}(0)\right)$ have a tangency of order $k$ at $\theta(0)$. Then, to study the $k$-jet of $R_{u, t}$ at $\theta(0)$ we can assume that $R_{u, t}$ leaves $\Sigma_{0}(0)$ invariant for all $t \in[0, a]$. 
Lemma 9. The $k$-jet at $\theta(0)$ of $\left.R_{u, t}\right|_{\Sigma_{0}(0)}$ is equal to the $k$-jet at $\theta(0)$ of the Hamiltonian flow at time $t$ that corresponds to the non autonomous Hamiltonian function $\left[\delta(t) F \circ\left(\left.\widehat{P}_{0, t}\right|_{\Sigma_{0}(0)}\right)\right]$ in $\Sigma_{0}(0)$.

Proof. Combining Proposition 7 and Remark 8, we conclude that the $k$-jet of $\left.R_{u, t}\right|_{\Sigma_{0}(0)}$ is equal to the $k$-jet of the flow at time $t$ associated to the field $\widehat{P}_{0, t}^{*}\left(Y_{u, t}\right)$. On the other hand, if $X$ denotes the Hamiltonian field for the non autonomous Hamiltonian $\left[K_{u, t} \circ\left(\left.\widehat{P}_{0, t}\right|_{\Sigma(0)}\right)\right]$, then, using that $\left.\widehat{P}_{0, t}\right|_{\Sigma(0)}$ : $\Sigma(0) \rightarrow \Sigma(t)$ is a symplectic map, we have:

$$
\begin{aligned}
\left.\omega(X, \cdot)\right|_{\Sigma(0)} & =d\left(K_{u, t} \circ\left(\left.\widehat{P}_{0, t}\right|_{\Sigma(0)}\right)\right)=\widehat{P}_{0, t}^{*}\left(d K_{u, t}\right)=\left.\widehat{P}_{0, t}^{*} \omega\left(Y_{u, t}, \cdot\right)\right|_{\Sigma(t)} \\
& =\left.\omega\left(\widehat{P}_{0, t}^{*}\left(Y_{u, t}\right), \cdot\right)\right|_{\Sigma(0)} .
\end{aligned}
$$

And, since $\left.\omega\right|_{\Sigma(0)}$ is no degenerate, we have that $X=\widehat{P}_{0, t}^{*}\left(Y_{u, t}\right)$. Hence the $k$-jet of $\widehat{P}_{0, t}^{*}\left(Y_{u, t}\right)$ at $\theta(0)$ is determined by the $(k+1)$-jet of the Hamiltonian $\left[K_{u, t} \circ\left(\left.\widehat{P}_{0, t}\right|_{\Sigma(0)}\right)\right]$ at $\theta(0)$, that, by (4), is equal to the $k$-jet of the Hamiltonian $\left[\delta(t) F \circ\left(\left.\widehat{P}_{0, t}\right|_{\Sigma(0)}\right)\right]$. This completes the proof.

Remark 10. Recall that $J_{s}^{k}(n)$ is a Lie group with the group structure defined by $j^{k} f \cdot j^{k} g=j^{k}(f \circ g)$. Let $\mathfrak{J}_{s}^{k}(n)$ be the space of the $k$-jets at 0 of symplectic vector fields that are zero at 0 . We define the bracket $[\cdot, \cdot]^{k}: \mathfrak{J}_{s}^{k}(n) \times \mathfrak{J}_{s}^{k}(n) \rightarrow$ $\mathfrak{J}_{s}^{k}(1)$ by $\left[j^{k} X, j^{k} Y\right]^{k}=-j^{k}[X, Y]$. Since $X, Y$ are zero in the origin, $[\cdot, \cdot]^{k}$ depends only on the $k$-jets of $X$ and $Y$. Then $[\cdot, \cdot]^{k}$ defines a Lie algebra structure in $\mathfrak{J}_{s}^{k}(n)$. Moreover, $\mathfrak{J}_{s}^{k}(n)$ is the Lie algebra of $J_{s}^{k}(n)$ and the exponential map exp : $\mathfrak{J}_{s}^{k}(n) \rightarrow J_{s}^{k}(n)$ verifies $\exp \left(t j^{k} X\right)=j^{k} \phi_{t}$, where $\phi_{t}$ is the local flow associated to $X$. For more details and proofs, see [KMS93, $\S \mathrm{IV]}$.

Definition 11. A one parameter family of symplectic maps $\sigma:[a, b] \rightarrow S p(n)$ is $k$-general if there exist times $t_{1}, \ldots, t_{d} \in[a, b]$ such that $\left(\sigma_{t_{1}}, \ldots, \sigma_{t_{d}}\right)$ is in $G_{k}$.

Recall the definition of the set $G_{k}$ in Section 2 .

Next, we show that it is possible to perturb the $k$-jet of the Poincaré map without changing its $(k-1)$-jet. To do this, let

$$
S_{a}^{k}: \mathcal{F}^{k} \rightarrow \operatorname{ker}\left(\pi_{k}\right) \subset J_{s}^{k}(n)
$$

be the map defined by

$$
S_{a}^{k}(u)=j^{k}\left(\left.R_{u, a}\right|_{\Sigma_{0}(0)}\right)(\theta(0))
$$

where $\pi_{k}: J_{s}^{k}(n) \rightarrow J_{s}^{k-1}(n)$ is the canonical projection. 
Proposition 12. If the one parameter family of linear symplectic maps

$$
\left.[0, a] \ni t \mapsto d_{\theta(0)} \widehat{P}_{0, t}\right|_{\Sigma_{0}(0)} \in S p(n),
$$

is $(k+1)$-general for some $k \geq 2$, then the map $S_{a}^{k}$ is a local submersion in a neighborhood of $0 \in \mathcal{F}^{k}$.

Proof. Let $d=d(2 n, k+1)$ be the dimension of the real vector space of homogeneous polynomials of degree $k+1$ in the $2 n$ variables $x=\left(x_{1}, \ldots, x_{n}\right)$ and $y=\left(y_{1}, \ldots, y_{n}\right)$. Since $\left.d_{\theta(0)} \widehat{P}_{0, t}\right|_{\Sigma(0)}$ is a $(k+1)$-general family of symplectic linear maps for $0 \leq t \leq a$, there are $t_{1}, \ldots, t_{d} \in(0, a)$, such that

$$
\left\{F\left(d \widehat{P}_{0, t_{1}}(x, y)\right), \ldots, F\left(d \widehat{P}_{0, t_{d}}(x, y)\right)\right\}
$$

is a basis of $\mathbb{R}_{k+1}[x, y]$, where $F(x, y)=x_{1}^{k+1} \in \mathbb{R}_{k+1}[x, y]$. For each $1 \leq i \leq d$ and $\lambda>0$ sufficiently small, let $\delta_{t_{i}}^{\lambda}: \mathbb{R} \rightarrow \mathbb{R}$ be a $C^{\infty}$ approximation of the Dirac delta function at the point $t_{i}$ with support in the interval $\left[t_{i}-\lambda, t_{i}+\lambda\right]$. We consider $u_{i} \in \mathcal{F}^{k}$ defined by

$$
u_{i}\left(x_{0}, x_{1}, \ldots, x_{n}\right)=\delta_{t_{i}}^{\lambda}\left(x_{0}\right) \beta\left(x_{1}, \ldots, x_{n}\right),
$$

for $i=1, \ldots, d$. By Lemma 9 and the properties of the exponential map, as recalled in Remark 10, we have that

$$
D_{0} S_{t}^{k} \cdot\left(u_{i}\right)=\left.\frac{\partial}{\partial s}\right|_{s=0} S_{t}^{k}\left(s u_{i}\right)=\left.\frac{\partial}{\partial s}\right|_{s=0} \exp \left(t j^{k}\left(s X_{i}\right)\right),
$$

where $X_{i}$ denotes the Hamiltonian field in $\Sigma(0)$ corresponding to the non autonomous Hamiltonian $\left[\delta_{t_{i}}^{\lambda}(t) F \circ\left(\left.\widehat{P}_{0, t}\right|_{\Sigma(0)}\right)\right]$. Computing the derivative with respect to $t$ in the above equality, we obtain:

$$
\begin{aligned}
\frac{d}{d t}\left(D_{0} S_{t}^{k} \cdot\left(u_{i}\right)\right) & =\left.\frac{\partial}{\partial s}\right|_{s=0}\left(\frac{\partial}{\partial t} \exp \left(t j^{k}\left(s X_{i}\right)\right)\right) \\
& =\left.\frac{\partial}{\partial s}\right|_{s=0}\left(d_{\left(t j^{k}\left(s X_{i}\right)\right)} \exp \cdot j^{k}\left(s X_{i}\right)\right) \\
& =\left.\frac{\partial}{\partial s}\right|_{s=0} j^{k}\left(s X_{i}\right)=j^{k} X_{i} .
\end{aligned}
$$

Then

$$
D_{0} S_{a}^{k} \cdot\left(u_{i}\right)=\int_{0}^{a} j^{k} X_{i} d t
$$

By definition of $X_{i}$ and (5), we have that, if $\lambda$ converges to 0 , then $D_{0} S_{a}^{k}$. $\left(u_{i}\right)$ converges to the $k$-jet at $\theta(0)$ of the Hamiltonian vector field in $\Sigma(0)$ corresponding to the autonomous Hamiltonian $H_{i}=\left[F \circ\left(\left.\widehat{P}_{0, t_{i}}\right|_{\Sigma(0)}\right)\right]$. Computing the $(k+1)$-jet of $H_{i}$ at $\theta(0)$, we obtain that

$$
j^{k+1} H_{i}=\left[F \circ\left(\left.d_{\theta(0)} \widehat{P}_{0, t_{i}}\right|_{\Sigma(0)}\right)\right] .
$$


Since $\left\{F \circ\left(\left.d_{\theta(0)} \widehat{P}_{t_{i}}\right|_{\Sigma(0)}\right): 1 \leq i \leq d\right\}$ is a basis for $\mathbb{R}_{k+1}[x, y]$, we have that, for $\lambda$ sufficiently small, $\left\{D_{0} S_{a}^{k} \cdot\left(u_{i}\right): 1 \leq i \leq d\right\}$ is a basis of the Lie algebra of the Lie subgroup $\operatorname{ker}\left(\pi_{k}\right)$. Hence the map $S_{a}^{k}$ is a local submersion.

Now, let $k_{0} \geq 1$ and $Q$, an open invariant subset of $J_{s}^{k_{0}}(n)$, be given as in the hypothesis of Theorem 2 . If we assume that the derivatives $\left.d_{\theta(0)} \widehat{P}_{0, t}\right|_{\Sigma_{0}(0)}$ are $k$-general for each $k=3, \ldots, k_{0}+1$, it follows from the proposition above that, for each $k=2, \ldots, k_{0}$, the map $S_{a}^{k}$ is an open map in a neighborhood of $0 \in \mathcal{F}^{k} \subset \mathcal{F}^{1}$. And this, together with the hypothesis $j^{k_{0}} P\left(\theta, \Sigma, H_{0}\right) \in \bar{Q}$, implies that there exists $u \in \mathcal{F}^{1}$ arbitrarily $C^{\infty}$-close to zero such that the $k_{0}$-jet of $\left.R_{u, a}\right|_{\Sigma_{0}(0)}$ is in $P^{-1} Q$. Then, $j^{k_{0}} P \in Q$.

Then to complete the proof of Theorem 2 , it only remains to verify that it is possible to perform a perturbation that makes the derivatives $\left.d_{\theta(0)} \widehat{P}_{0, t}\right|_{\Sigma_{0}(0)}$ $k$-general, for each $k=3, \ldots, k_{0}+1$. This is the content of the next lemma.

Lemma 13. For each integer $k>2$, there exists a smooth potential $u: M \rightarrow$ $\mathbb{R}$, arbitrarily close to 0 in the $C^{\infty}$-topology, such that $\theta(t)$ is also a closed orbit of the Hamiltonian flow of $H_{u}$ and the one parameter family $t \mapsto d_{\theta(0)} P_{u, t}$, for $t \in[0, a]$, is k-general.

Proof. Consider the map $S_{t}^{1}: \mathcal{F}^{1} \rightarrow S p(n)$ defined by

$$
S_{t}^{1}(u)=d_{\theta(0)} P_{u, t}
$$

Given $k \geq 2$, let $d=d(2 n, k+1)$ be the dimension of the real vector space of homogeneous polynomials of degree $k+1$ in the $2 n$ variables $x=\left(x_{1}, \ldots, x_{n}\right)$ and $y=\left(y_{1}, \ldots, y_{n}\right)$. Choosing times $0<t_{1}<\cdots<t_{d}<a$ we define the map

$$
\Phi: \mathcal{F}^{1} \rightarrow S p(n)^{d}
$$

given by

$$
\Phi(u)=\left(S_{t_{1}}^{1}(u), \ldots, S_{t_{d}}^{1}(u)\right) .
$$

Then, using Proposition 4.2 and Lemma 4.3 in [RR11], we obtain that each component of the map $\Phi$ is a local submersion near $0 \in \mathcal{F}^{1}$. Since $G_{k} \subset$ $S p(n)^{d}$ is dense (Proposition 6), we can choose a potential

$$
u=u_{1}+\cdots+u_{d} \in \mathcal{F}^{1}
$$

with $\operatorname{supp}\left(u_{i}\right) \cap \gamma\left(\left[t_{j-1}, t_{j}\right]\right)=\emptyset$ for $i \neq j$, arbitrarily close to 0 in the $C^{\infty}$ topology, and such that the one parameter family of linear symplectic maps associated to the linearized Poincaré map of the perturbation $H_{u}$ is $k$-general. 


\section{Proof of Theorem 3}

Let $Q$ be an open, dense and invariant subset of $J_{s}^{k}(n)$ and $c \in \mathbb{R}$. As in the Kupka-Smale Theorem, for each $m \in \mathbb{N}$, let $\mathcal{G}(m) \subset C^{\infty}(M)$ be such that $H_{u}^{-1}(c)$ are regular and every closed orbit of the Hamiltonian flow of $H_{u}$ in $H_{u}^{-1}(c)$ of period less than or equal to $m$ are non-degenerate, for all $u \in \mathcal{G}(m)$. Then $\mathcal{G}(m)$ is an open and dense subset of $C^{\infty}(M)$ with the $C^{\infty}$-topology (see Lemma 3.3 in [Oli08]). Let $\mathcal{O}(m) \subset \mathcal{G}(m)$ be the set of $C^{\infty}$-potentials $u: M \rightarrow \mathbb{R}$ such that the $k$-jet of the Poincaré map of every closed orbit of the Hamiltonian flow of $H_{u}$ in $H_{u}^{-1}(c)$ of period less than or equal to $m$ belongs to $Q$. Since the set of periodic orbits of the Hamiltonian flow of $H_{u}$ in $H_{u}^{-1}(c)$ of period less than or equal to $m$ is finite, for all $u \in \mathcal{O}(m)$, and by continuity of the Poincaré map, we have that $\mathcal{O}(m)$ is open. By Theorem $2, \mathcal{O}(m)$ is also a $C^{\infty}$-dense subset of $C^{\infty}(M)$. Therefore

$$
\mathcal{O}=\bigcap_{m \in \mathbb{N}} \mathcal{O}(m)
$$

is the residual subset that we were looking for.

\section{ACKNOWLEDGMENTS}

We are grateful to G. Contreras and M. J. Dias Carneiro, for the helpful conversations. We also thank the referee, who kindly pointed out a few mistakes.

\section{REFERENCES}

[]Abr70 R. Abraham, Bumpy metrics, Global Analysis (Proc. Sympos. Pure Math., Vol. XIV, Berkeley, Calif., 1968), Amer. Math. Soc., Providence, R.I., 1970, pp. 1-3. MR 0271994 (42 \#6875)

[]Ano82 D. V. Anosov, Generic properties of closed geodesics, Izv. Akad. Nauk SSSR Ser. Mat. 46 (1982), no. 4, 675-709, 896. MR 670163 (84b:58029)

[]BC08 P. Bernard and G. Contreras, A generic property of families of Lagrangian systems, Ann. of Math. (2) 167 (2008), no. 3, 1099-1108. MR 2415395 (2009d:37113)

[1CI99 G. Contreras and R. Iturriaga, Convex Hamiltonians without conjugate points, Ergodic Theory Dynam. Systems 19 (1999), no. 4, 901-952. MR 1709426 (2000h:37102)

[]Con10 G. Contreras, Geodesic flows with positive topological entropy, twist maps and hyperbolicity, Ann. of Math. (2) $\mathbf{1 7 2}$ (2010), no. 2, 761-808. MR 2680482

[]CP02 G. Contreras and G. P. Paternain, Connecting orbits between static classes for generic Lagrangian systems, Topology 41 (2002), no. 4, 645-666. MR 1905833 (2003i:37059)

[]Kli78 W. Klingenberg, Lectures on closed geodesics, Springer-Verlag, Berlin, 1978, Grundlehren der Mathematischen Wissenschaften, Vol. 230. MR 0478069 (57 \#17563)

[]KMS93 I. Kolářr, P. W. Michor, and J. Slovák, Natural operations in differential geometry, Springer-Verlag, Berlin, 1993. MR 1202431 (94a:58004)

[]KT72 W. Klingenberg and F. Takens, Generic properties of geodesic flows, Math. Ann. 197 (1972), 323-334. MR 0307282 (46 \#6402) 
[]Mañ96 R. Mañé, Generic properties and problems of minimizing measures of Lagrangian systems, Nonlinearity 9 (1996), no. 2, 273-310. MR 1384478 (97d:58118)

[]Mas03 D. Massart, On Aubry sets and Mather's action functional, Israel J. Math. 134 (2003), 157-171. MR 1972178 (2004g:37088)

[]Mat91 J. N. Mather, Action minimizing invariant measures for positive definite Lagrangian systems, Math. Z. 207 (1991), no. 2, 169-207. MR 1109661 (92m:58048)

[]Mir06 J. A. G. Miranda, Generic properties for magnetic flows on surfaces, Nonlinearity 19 (2006), no. 8, 1849-1874. MR 2250797 (2007f:37094)

[]Oli08 E. Oliveira, Generic properties of Lagrangians on surfaces: the Kupka-Smale theorem, Discrete Contin. Dyn. Syst. 21 (2008), no. 2, 551-569. MR 2385706 (2009b:37103)

[]RR11 L. Rifford and R. Ruggiero, Generic properties of closed orbits for Hamiltonian flows from Mañé's viewpoint, Int. Math. Res. Not. IMRN, to appear. doi:10.1093/imrn/rnr231

Universidade Federal de Minas Gerais, Av. Antônio Carlos 6627, 31270-901, Belo Horizonte, MG, Brasil.

E-mail address: carballo@mat.ufmg.br

Universidade Federal de Minas Gerais, Av. Antônio Carlos 6627, 31270-901, Belo Horizonte, MG, Brasil.

E-mail address: jan@mat.ufmg.br 